\title{
Food matrix and isoflavones bioavailability in early post menopausal women: A European clinical study
}

\author{
Brigitte Chanteranne ${ }^{1,2}$ \\ Francesco Branca ${ }^{3}$ \\ A Kaardinal ${ }^{4}$ \\ K Wahala ${ }^{5}$ \\ Véronique Braesco 6 \\ Philippe Ladroite ${ }^{7}$ \\ Fred Brouns ${ }^{8}$ \\ Véronique Coxam ${ }^{1,2}$ \\ 'INRA, Centre Clermont-Ferrand - \\ Theix, UMR I019, Unité Nutrition \\ Humaine, St Genès Champanelle, \\ France; ${ }^{2}$ Univ Clermont I, UFR \\ Médecine, UMRI019, Unité Nutrition \\ Humaine, Clermont-Ferrand, \\ France; ${ }^{3}$ INRAN, Human Nutrition \\ Unit, Ardeatina, Rome, Italy; ${ }^{4} \mathrm{TNO}$ \\ Nutrition and Food Research, Zeist, \\ The Netherlands; ${ }^{5}$ Department of \\ Chemistry, University of Helsinki, \\ Helsinki, Finland; 'Danone Vitapole, Le \\ Plessis Robinson, France; ${ }^{7}$ Nutrition \\ Santé, Ravel, France; ${ }^{8}$ Eridania \\ Beghin Say, Vilvoorde Research and \\ Development Centre, Vilvoorde, \\ Belgium
}

\begin{abstract}
The estrogenic effects of soy isoflavones (IF) on symptoms of menopause are of particular interest. The aim of the present study was to improve compliance of IF in two IF-enriched foods providing the same IF circulating levels in postmenopausal women. Forty-two healthy postmenopausal women (mean age: 53.28 years) were recruited for a randomized, crossover, multicenter trial conducted in the Netherlands, Italy and France. Over 18 days, volunteers were assigned to two groups and supplemented with two different IF-enriched foods (100 mg IF aglycones/two servings). The first group had to eat two biscuits daily for three days. After a wash-out period $(11 \mathrm{~d})$, they received cereal bars for three days. The second group started with the cereal bars and finished with biscuits. After IF intake, plasma and urinary levels of genistein, daidzein, $\mathrm{O}$ desmethyl angolensin and equol significantly increased and returned to baseline level after the washout period. There was no difference between biscuits and cereals bars intake, as shown by group values at each end of experimental period (day 4 or day 18). Both matrixes are comparable in terms of IF-circulating levels and could be used independently.
\end{abstract}

Keywords: soy isoflavones, bioavailability, food processing, postmenopausal women, equol

\section{Introduction}

Current interest in soy foods is largely driven by documented research on the potential health benefits of phytoestrogens (Nomura et al 1978; Anderson et al 1995; Knight and Eden 1995). Soybeans are a staple of the Asian diet (Messina 1995). The main isoflavone exposure is $17.7 \mathrm{mg}$ total isoflavones (IF) per day in China (Lui et al 2004) and $47.2 \mathrm{mg}$ per day total IF in Japan (Arai et al 2000). In contrast, the estimated mean dietary daily IF intake reported for the UK was calculated to be $0.8 \mathrm{mg}$ (Jones et al 1989). The average consumption of phytoestrogens in Europe is estimated to be less than $2 \mathrm{mg}$ /day (Boker et al 2002; Van Erp-Baart et al 2003). Consequently, an increase in soy consumption in Western countries could be a promising innovative target. A positive relationship has been demonstrated between the level of soy consumption and bone mineral density (Potter et al 1998). However, what is critical to biological efficacy of these compounds is a thorough understanding of their bioavailability from different soya foods (Pascual-Teresa et al 2006). Indeed, the composition of the IF glucoside conjugates, which is altered during the industrial processing and cooking (Coward et al 1998), should modify the rate of absorption and possibly the degree of further metabolism.

Futhermore, the variability of IF bioavailability can also be due to inter-individual differences in IF metabolism (Rowland et al 2003). As a matter of fact, only 20\%-35\% of the adult population is able to convert daidzein into equol after ingesting soy or soy derivative products (Setchell et al 2005). This conversion is carried out by the intestinal bacteria (Setchell et al 1984; Atkinson et al 2005). These inter-individual differences could, at least, partially explain the differences in the biological effects 
observed following soy consumption. In a 2-year study carried out in post-menopausal women who drank $500 \mathrm{ml}$ of soymilk either with or without IF, greater effects on bone health were elicited when volunteers were able to produce equol (Lydeking-Olsen et al 2002).

This study is the first step of an interventional multicenter trial which aimed to study the potential preventive effect of soy IF on bone mass in postmenopausal women (PHYTOS shared-cost European RTD program: the prevention of osteoporosis by nutritional phytoestrogens, QLK1-CT2000-00431). The purpose of this work package was to set up a pilot trial to improve compliance of IF in two IF-enriched foods providing the same IF circulating levels in postmenopausal women.

\section{Materials and methods Study design}

The study was a randomized, crossover, parallel multicenter trial conducted in the Netherlands (TNO Quality of Life, Zeist), Italy (INRAN, Rome), and France (INRA, ClermontFerrand). The protocol of this study was approved by the ethical committee in each country (the independent Medical Ethics Committee of TNO for the Netherlands, The Medical Ethics Committee of the University of Rome "La Sapienza" for Italy and the "Comité Consultatif de Protection des Personnes dans la Recherche Biomédicale" for France).

Subjects were healthy Caucasian females selected via a phone questionnaire. All the volunteers gave their written informed consent. In each country, 14 healthy ambulatory postmenopausal women, less than five years and menstrual bleeding within 12 months, (mean age: 53.28, range from 49 to 60 years; mean age at menopause: 50.78 years) were recruited and completed the study. Subjects had a body mass index (BMI) ranging from 20 to $29 \mathrm{~kg} / \mathrm{m}^{2}$. Subjects were randomly allocated to two group with BMI, age and time since last menses as randomization variables.

Subjects osteoporotic, with severe scoliosis, with a history of medical or surgical events that might significantly affect the study outcome, or who were using a concomitant medication known to affect bone metabolism were excluded. Interfering habits such as strict vegetarian and vegan, high fiber and high soy diets, allergy for soy, and frequent alcohol consumption ( $>2$ drinks/day, $>15$ drinks/week) were considered as exclusion criteria, as well. Participants were willing to take soy-enriched products, had not been involved in any clinical trial six months before entering this study and, were not in the exclusion period of the national index of healthy volunteers.
Volunteers underwent a screening visit that included a health history questionnaire, a physical examination and routine blood chemistry. The nutrition history questionnaire was used to investigate usual dietary intake patterns.

During the whole study (18 days), volunteers were requested to consume their habitual diet, although they were given detailed dietary instructions to minimize prebiotics-containing food products or to avoid phytoestrogen consumption. Subjects were assigned to one of two groups (seven women each). Two different IF-enriched foods were provided to the volunteers: biscuits (Danone Vitapole, Palaiseau France) and cereals bars (Nutrition Santé, Revel, France). They both were designed to bring $100 \mathrm{mg}$ IF aglycones in two servings (60\%-75\% genistein; $23 \%-35 \%$ daidzein; $1 \%-5 \%$ glycitin; 83.5 mg IF glycosyled; Prevastein ${ }^{\circledR} \mathrm{HC}$, Eridania Beghin-Say, Vilvoorde, Belgium). The composition is given in Table 1. The first group of volunteers had to eat, including meals, two IF-enriched biscuits daily for three days (D1-D3). After a wash-out period of 11 days (D4-D14), under the same conditions they were provided two IF-enriched cereal bars for three days (D15-D18). The second group started with the cereal bars and received the biscuits during the second period (Figure 1).

Compliance with consumption was monitored by a combination of specially designed forms and return of unconsumed foods throughout the study and the assessment of plasma and urinary IF concentrations at four time points.

\section{Sample size calculation}

The sample size has been calculated to detect differences in plasma levels of total IF between groups consuming different food matrixes equal to $1 / 2 \mathrm{SD}(0.15 \mu \mathrm{mol} / \mathrm{L})$ observed in 12 adult women after a single dose of $126 \mathrm{mg}$ IF administered as soy milk (Xu et al 1994). A total of 42 subjects (14 per country) is required to detect differences between groups with $80 \%$ power, significance level set at 0.05 , and assuming a $20 \%$ drop-out rate.

\section{Blood and urine collection}

At days 1, 4, 15, and 18, $7 \mathrm{ml}$ of blood were collected by venous puncture, ie, a total volume of $28 \mathrm{ml}$. The volunteers were asked to fast since their dinner on the previous day. Venous blood samples were drawn on heparin tubes subjected to centrifugation $\left(2000 \mathrm{~g} \times 10 \mathrm{~min}\right.$ at $\left.4{ }^{\circ} \mathrm{C}\right)$. Then plasma was aliquoted and frozen $\left(-80^{\circ} \mathrm{C}\right)$ for genistein and daidzein analysis and one was archived. 
Table I Composition of the investigational products per $100 \mathrm{~g}$ of product ${ }^{1}$

\begin{tabular}{lll}
\hline Nutritional analysis & Biscuits & Bars \\
\hline Energy (Kj) & 1846 & 1600 \\
Moisture (g) & 2.6 & 8.6 \\
Protein (g) & 6.6 & 6.0 \\
Carbohydrates (g) & 64.3 & 77.9 \\
Sugars (g) & 26.3 & 24.1 \\
Fat (g) & 17.3 & 4.4 \\
Saturated fatty acids (g) & 4.3 & 2.2 \\
Monounsaturated fatty acids (g) & 8.9 & 0.2 \\
Polyunsaturated fatty acids (g) & 4.0 & 1.5 \\
Fibers (g) & 6.6 & 1.7 \\
Isoflavones aglycones (mg) & 444 & 254 \\
Minerals & & \\
Calcium (mg) & 21 & 15 \\
Magnesium (mg) & 36 & 40 \\
Phosphorus (mg) & 131 & 103 \\
Iron (mg) & 1.22 & 1.03 \\
\hline
\end{tabular}

Note: 'The subjects received combinations of the products equalling $24 \mathrm{~g} / \mathrm{d}$ biscuits or $12 \mathrm{~g} / \mathrm{d}$ bars.

Subjects were also required to complete a 24-h urine samples collection before each visit (from $7 \mathrm{am}$ the previous day to 7 am the visit day) on days $1,4,15$, and 18 and brought back the flask. The volume of each 24-h urine sample was measured, and subsamples were taken, adding $0.1 \%$ ascorbic acid and frozen $\left(-20{ }^{\circ} \mathrm{C}\right)$ until genistein, daidzein, equol and O-DMA analysis. One aliquot was archived.

\section{Isoflavone assessment}

Genistein and daidzein were analyzed, in duplicate in plasma, by RIA (TR-FIA), using as internal standard tritiated estradiol beta-glucuronide. The sensitivity limit of the method is $1.8 \mathrm{pg} / \mu 1,0.35 \mathrm{nM}$, being recovery calculated after hydrolysis and extraction.

Genistein, daizein, equol and O-DMA were analyzed in duplicate in urine sample by GC/SIM, using deuterium labeled internal standards. In this method, isoflavonoids were hydrolyzed and measured as free aglycones. This newly-developed immunoassay for IF was compared with GC/MS and HPLC methods to ensure adequate performance in terms of their precision, specificity and spiking/recovery of added synthetic isoflavones (Adlercreutz et al 2004).

\section{Statistics}

The data are expressed as individual values and means \pm standard error of the mean (SEM). Statistical tests have been chosen according to the data distribution (KolmorogovSmirnov test). For each group a comparison was established between results at the first experimental day and after three days of consumption. Analysis of variance (ANOVA) was used to test for any significant difference among groups. These tests were carried out under Statview software (Microsoft, San Diego, CA, USA). A two-way ANOVA was performed, one way for the country and the other way for the treatment. When ANOVA was significant the Student's T-test was used.

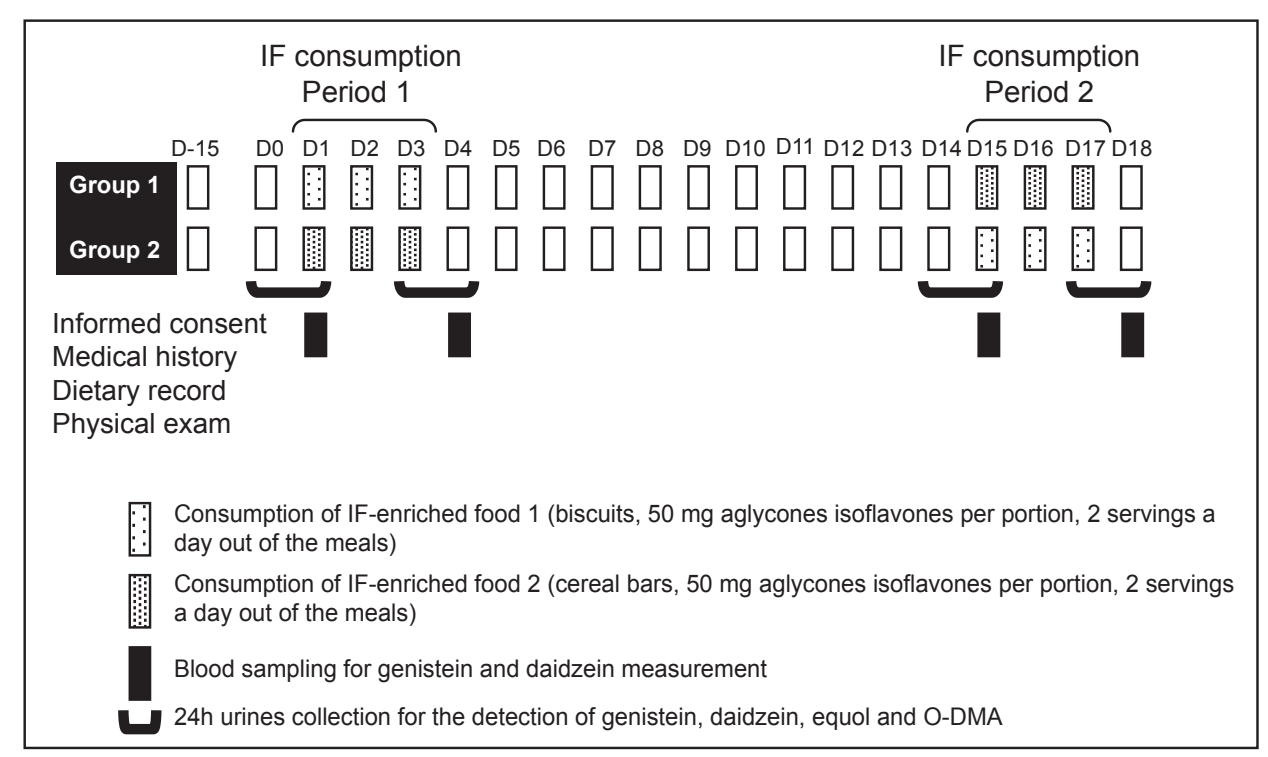

Figure I Study design to test the effect of food matrix on isoflavones bioavailability in early postmenopausal women. 


\section{Results}

\section{Effect of food matrix on isoflavones bioavailability}

In France, urinary and plasma levels of genistein and daidzein followed the same pattern, ie, an increase at day 4 and 18 (Figure 2). Basal plasma genistein levels at day 1 were not significantly different from those measured at the beginning of the second experimental period (day 15). Daidzein concentrations followed the same pattern. Consequently, the wash-out period was efficient. Whatever the IF consumption period, plasma concentrations increased after 3 days and values reached similar levels at day 4 and day 18 .
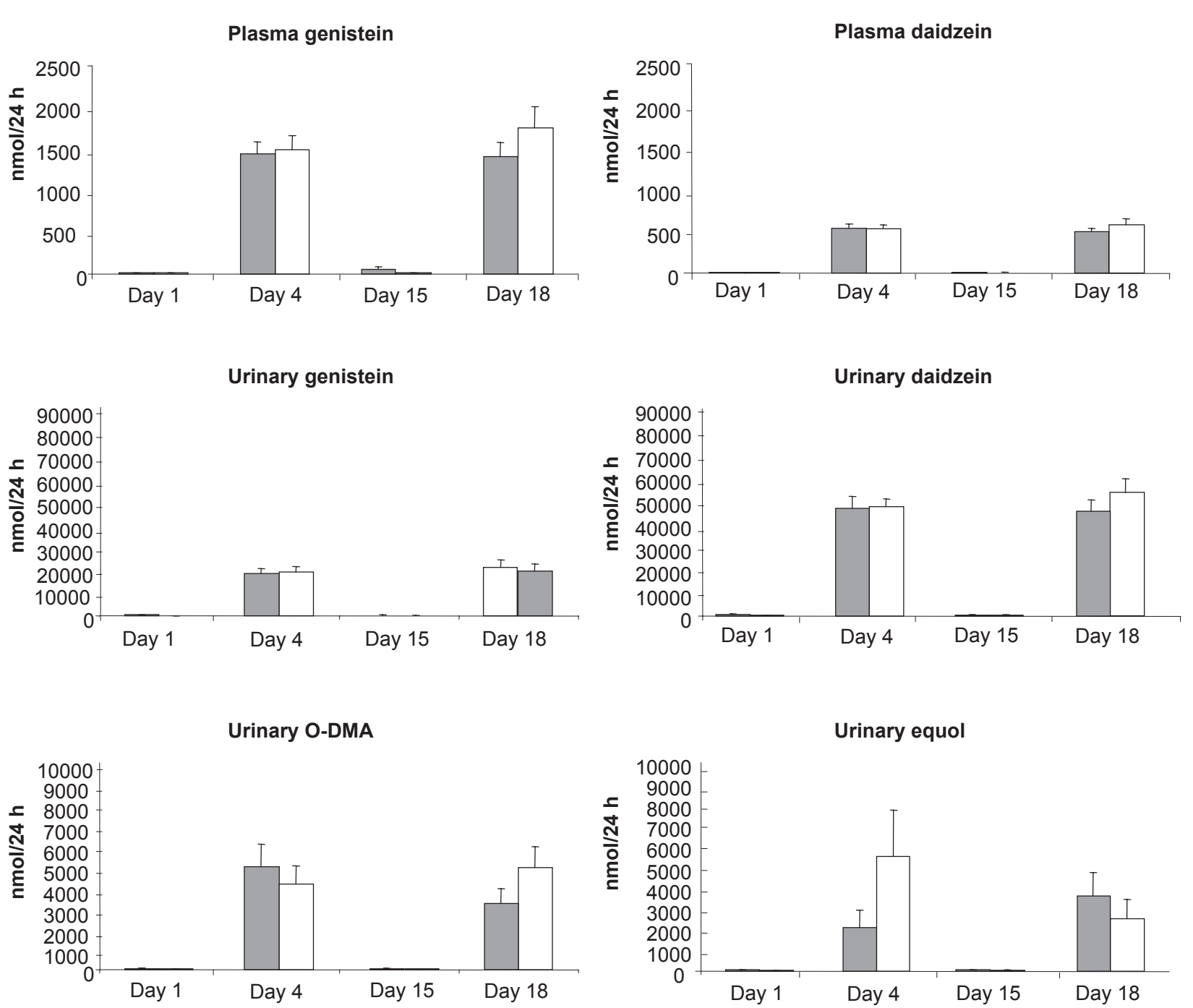

The two food matrix did not have any influence on equol productions, as shown by the number of equol producers and non producers (Figure 3 ).

\section{Isoflavones bioavailability in each country}

Whatever the period, plasma genistein levels did not differ among volunteers in each center. The highest basal levels of

Figure 2 Effect of isoflavones-enriched biscuits $(\square)$ or isoflavones-enriched cereal bars ( $\square$ ) consumption for 3 days (day I to day 4 and day I5 to day I8) on plasma genistein and daidzein levels, and urinary genistein, daidzein, equol and O-DMA concentrations. Values are expressed as means \pm their standard errors (values for each country have been plotted together). Means values were all significantly different from day I or day 15. 


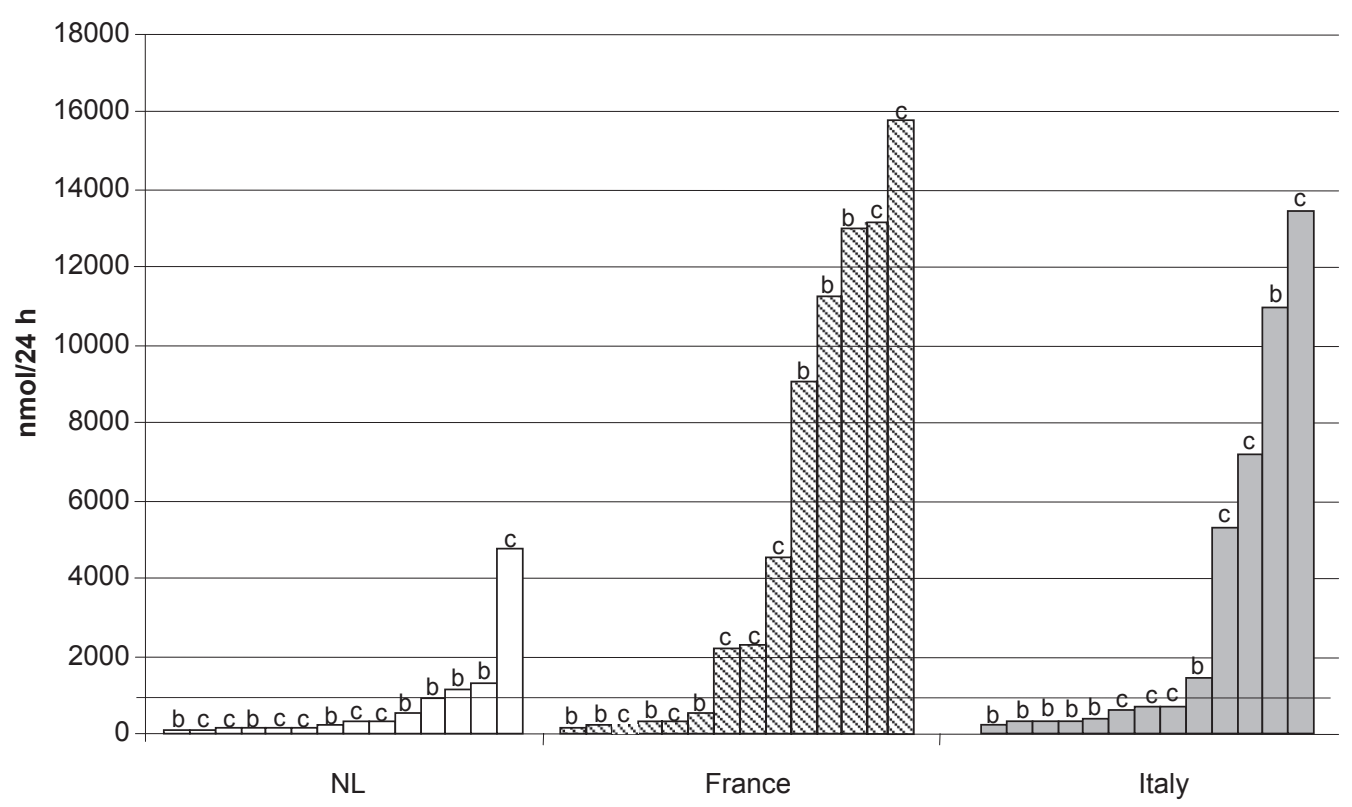

Figure 3 Effect isoflavones-enriched biscuits (b) or isoflavones-enriched cereal bars (c) consumption for 3 days (day I5 to day I8) on urinary equol excreation at day I8. Individual values are given in each country (the Netherlands $(\square)$, France ( $\mathrm{Q}$ ) and italy $(\square)$, values above the dotted line (at $1000 \mathrm{nmol} / 24 \mathrm{~h}$ ) were harvested in volunteers considered as high producers.

daidzein were measured in Netherlands and being even lower in France than in Italy. After three days of IF consumption, daidzein values were similar in all three countries except at day 4 in Italy.

In Netherlands, genistein urinary excretion was lower $(\mathrm{p}<0.05)$ than in France and in Italy at day 4 and 18. Daidzein urinary excretion was lower $(\mathrm{p}<0.05)$ than those in Italy (Figure 4).

Average equol excretion rate (nmol/24 h) was much lower in the Netherlands than in the other countries (France: $5212 \pm 1268$; Italy: $3218 \pm 1268$ ). In the Netherlands, only $28.6 \%$ of the volunteers were considered as high equol producers ( $>1000 \mathrm{nmol} / 24 \mathrm{~h})$, against $57 \%$ in France and the average urinary production among those producers (nmol/24 h) was much lower than in the other countries (Table 2).

\section{Discussion}

Following soy food consumption, IF are biotransformed by intestinal microflora, a required step for absorption. IF are hydrolyzed by glucosidases to the aglycones daidzein, genistein and glycitein. Some are further metabolized to compounds such as equol, dihydrodaidzein and p-ethylphenol. Equol is more estrogenic than its precursors, whereas, p-ethylphenol is nonestrogenic. Daidzein is formed from formononetin and is metabolized into equol and O-DMA. Genistein is formed from biochanin A and metabolised to p-ethylphenol. Elimination of IF is predominantly via the urine (mainly equol), although total recovery by mass balance is less than $50 \%$, suggesting that unrecognized metabolites are being formed. Only some pharmacokinetic studies have been conducted in which isoflavones were measured in human subjects simultaneously in plasma urine and feces (Xu et al 1994). Hutchins and colleagues (1995) determined the metabolites O-DMA and equol, in addition to genistein and daidzein in urine after intake of fermented and unfermented soy foods, but no analysis was performed in blood.

In the present study, IF plasma levels would reach a plateau after three days of IF-enriched food consumption and would return to baseline after a wash-out period of one week. A significant increase in both plasma and urinary IF was shown after three days of consumption and concentrations returned to basal level in 15 days (wash-out period). This pattern was demonstrated to be similar in each of the three countries. According to data at the time the experiment started, elimination half-lifes of genistein and daidzein were considered to be short: 8 to $10 \mathrm{~h}$ and 6 to $8 \mathrm{~h}$ for genistein and daidzein, respectively. Moreover, several studies demonstrated entero-hepatic recirculation phenomena 
Plasma genistein

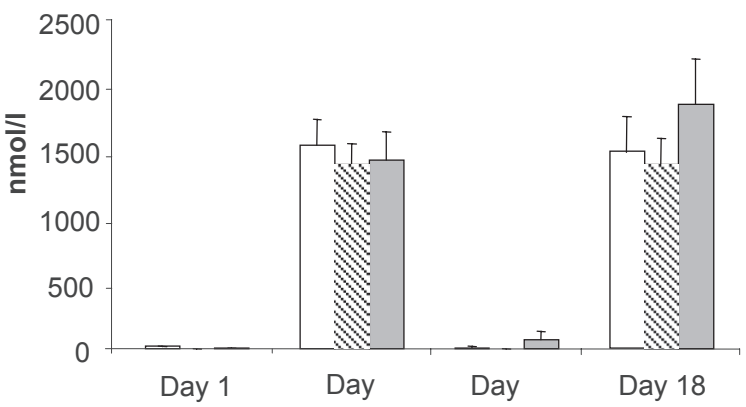

Urinary genistein

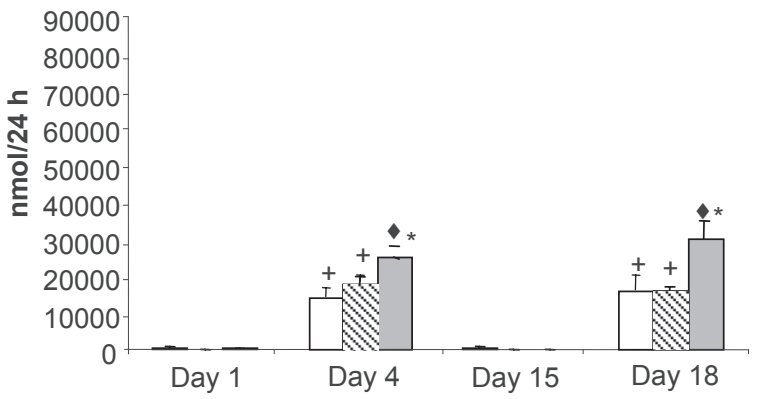

Urinary O-DMA

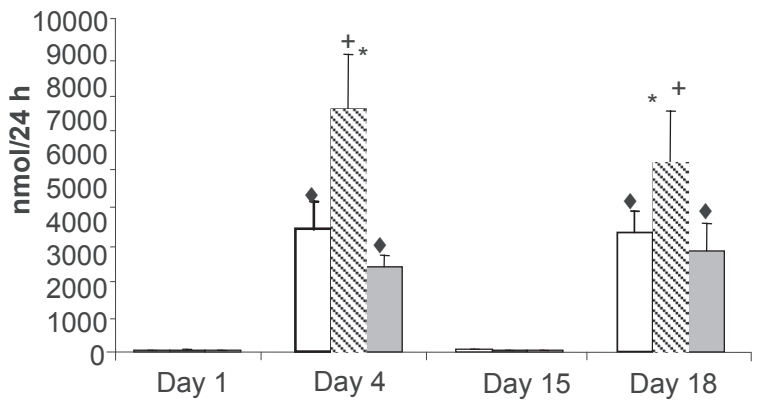

$\square$ The Netherlands $\quad$ \& France $\quad \square$ Italy
Plasma daidzein

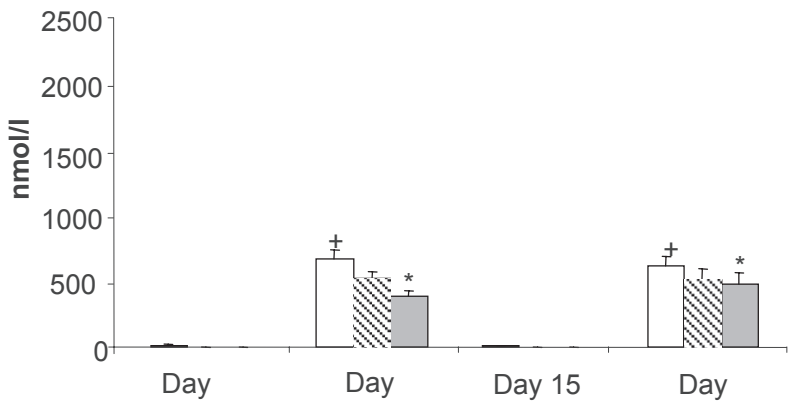

Urinary daidzein

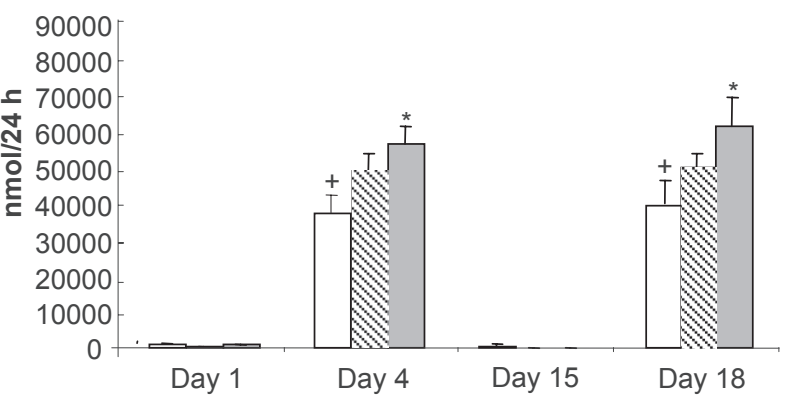

Urinary equol

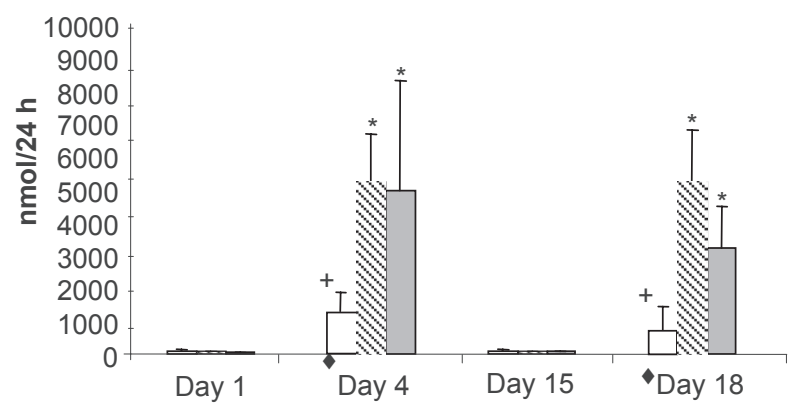

Figure 4 Effect of isoflavones-enriched food consumption for three days (day I to day 4 and day 15 to day 18) on plasma genistein and daidzein levels, and urinary genistein, daidzein, equol and O-DMA concentrations in the Netherlands ( $\square$ ), France ( $\mathrm{Q}$ ) and Italy $(\square)$.

Notes: Values are expressed as means \pm their standard errors. Values for both biscuits and cereal bars have been plotted together. Means values were all significantly different from day I or day 15. ${ }^{\mathrm{a}} \mathrm{P}<0.05$ vs concentrations in France; ${ }^{*} \mathrm{p}<0.05$ vs concentrations in The Netherlands, ${ }^{+} \mathrm{p}<0.05$ vs concentrations in Italy.

which lead to rather higher parameters up to $20 \mathrm{~h}$ for both IF (Richelle et al 2002; Vergne et al 2007).

In the present study plasma level reached quite high levels after consumption of IF-enriched biscuits or IF-enriched bars (nmol/1 magnitude). In addition, because Prevastein contains more genistein (167 mg IF glycosyled/day composed of $100-125 \mathrm{mg}$ genistein, $42-58 \mathrm{mg}$ daidzein and 1.67-8.35 mg glycitin), the higher genistein concentrations than daizein make sense. In each country, daidzein concentrations in plasma were lower than those of genistein which reflect the ingested proportions and the reverse was observed in the urine samples. This is in accordance with previous studies undertaken on humans and rats (Setchell et al 2003; Mathey et al 2006) showing that daidzein excretion is higher than that of genistein. This phenomenon reflects a greater bioavailability of daidzein.

Several investigators have reported that individual variability in colonic microflora plays an important role in determining the preferred pathways of isoflavones metabolism and the bioavailability of isoflavones. There is variation in the ability of the bacteria to produce equol, about $30 \%-50 \%$ of the adult population does not excrete 
Table 2 Ability to produce equol in The Netherlands, France and Italy

\begin{tabular}{llll}
\hline & The Netherlands & France & Italy \\
\hline Non equol producers $(<500 \mathrm{nmol} / 24 \mathrm{~h})$ & $64.3 \%$ & $28.6 \%$ & $53.8 \%$ \\
Low equol producers $(>500 \mathrm{nmol} / 24 \mathrm{~h})$ & $7 \%$ & $14 \%$ & $15.4 \%$ \\
High equol producers $(>1000 \mathrm{nmol} / 24 \mathrm{~h})$ & $28.6 \%$ & $57 \%$ & $30.8 \%$ \\
Average production in high producers $(\mathrm{nmol} / 24 \mathrm{~h})$ & $240 \mathrm{I} \pm\left. 84\right|^{\mathrm{a}}$ & $8906 \pm 1765$ & $767 \mid \pm 1933$ \\
\hline
\end{tabular}

Note: ${ }^{\mathrm{a}} \mathrm{P}<0.05$ vs values in France or Italy.

this bacterial metabolite in urine, when challenged daily with soy foods (Lampe et al 1998; Rowland et al 1999). In the early studies, it was found that two third of adults who consumed soy containing foods converted daidzein to equol (Setchell et al 1984). This ability to converted daidzein into equol led to use of the term "converters" to describe persons who have the necessary bacterial enzymes or intestinal conditions to make this biotransformation (Setchell et al 2001). In the present study, we have shown a significant difference in the ratio for equol producers in the three countries. IF was classified in three groups; according to the magnitude of production: high, medium and very low, French volunteers had the most important percentage of high equol producers (42\%), in opposite to Italy $(30 \%)$ or the Netherlands $(21 \%)$. The very low producers were much numerous in The Netherlands and as volunteers exhibited quite important individual variations in basal plasma IF (including genistein and daidzein). Consequently, it can be hypothesized that country differences may be explained by dietary factors. This trend will have to be checked in the main trial on more postmenopausal women.

In this project, a difference in composition of product, in particular in fiber level $(6.6 \mathrm{~g} / 100 \mathrm{~g}$ of biscuits vs $3.4 \mathrm{~g} / 100 \mathrm{~g}$ of bars) would have been modified the bioavailability of IF. Indeed, it has been established that concurrent dietary intake, in particularly fibers, may exert a major influence on isoflavones metabolism, by modulating intestinal microflora (Xu et al 1995). However, the level of IF did not appear to be altered by food matrix.

In conclusion, because the food matrix of biscuit or cereal bar does not modify IF metabolism, volunteers in the main intervention trial will have a choice between the biscuit or cereal bar.

\section{Acknowled-gments}

The authors acknowledge the collaboration of Paola D'Acapito, Valentina Di Mattei, Silvia Valtuena and MarieJeanne Davicco in the implementation of the clinical trial.
The study was carried out within the project PHYTOS $n^{\circ}$ QLK1-CT-2000-00431. This study does not necessarily reflect the views of the commission and in no way anticipates the Commission's future policy in this area. The authors report no conflicts of interest.

\section{References}

Adlercreutz H, Kiuru P, Rasku S, et al. 2004. An isotope dilution gas chromatographic-mass spectrometric method for the simultaneous assy of estrogens and phytoestrogens in urine. J Steroid Biochem Mol Biol, 92:399-411.

Anderson JW, Johnstone BM, Cook-Newell ME. 1995. Meta-analysis of the effects of soy proten intake on serum lipids. N Eng $J$ Med, 333:276-82.

Arai Y, Watanabe S, Kimira M, et al. 2000. Dietary intakes of flavonols, flavones and isoflavones by Japanese women and the inverse correlation between quercetin intake and plasma LDL cholesterol concentration. J Nutr, 130:2243-50.

Atkinson C, Frankenfeld CL, Lampe JW. 2005. Gut bacterial metabolism of the soy isoflavone daidzein: exploring the relevance to human health. Exp Biol Med (Maywood), 230:155-70.

Boker L K, van der Schouw YT, de Kleijn MJ, et al. 2002. Intake of dietary phytoestrogens by Dutch women. J Nutr, 132:1319-28.

Coward L, Barnes NC, Setchell KD, et al. 1993. Genistein, daidzein and their b-glycoside conjugates: antitumor isoflavones in soybean foods from American and Asian diets. J Agric Food Chem, 41:1961-7.

De Pascual-Teresa S, Hallund J, Talbot D, et al. 2006. Absorption of isoflavones in humans: effects of food matrix and processing. J Nutr Biochem, 17:257-64.

Hutchins AM, Slavin JL, Lampe JW. 1995. Urinary isoflavonoid phytoestrogen and lignan excretion after consumption of fermented and unfermented soy products. J Am Diet Assoc, 95:545-51.

Jones AE, Proce KR, Fenwick GR. 1989. Development and application of a high-performance liquid chromatographic method for the analysis of phytoestrogens. J Sci Food Agric, 46:357-64.

Knight DC, Eden JA. 1995. Phytoestrogens-a short review. Maturitas, 22:167-75.

Liu Z, Li W, Sun J, et al. 2004. Intake of soy foods and soy isoflavones by rural adult women in China. Asia Pac J Clin Nutr, 13:204-9.

Lydeking-Olsen E, Jensen JBE, Setchell KDR, et al. 2002. Isoflavone-rich soymilk prevents bone-loss in the lumbar spine of postmenopausal women. A 2 year study. $J$ Nutr, 132:581S.

Mathey J, Lamothe V, Coxam V, et al. 2006. Concentrations of isoflavones in plasma and urine of post-menopausal women chronically ingesting high quantities of soy isoflavones. J Pharm Biomed Anal, 41:957-65.

Messina M. 1995. Modern application for an ancient bean: soybeans and the prevention and treatment of chronic disease. J Nutr, 125:567S-9S.

Nomura A, Henderson BE, Lee J. 1978. Breast cancer and diet among the Japanese in Hawaii. Am J Clin Nutr, 31:2020-5.

Potter SM, Baun JA, Teng H, et al. 1998. Soy protein and isoflavones: their effects on blood lipids and bone density in postmenopausal women. Am J Clin Nutr, 68:1375-9. 
Richelle M, Pridmore-Merten S, Bodenstab S, et al. 2002. Hydrolysis of soflavone glycosides to aglycones by beta-glycosidase does not alter plasma and urine isoflavone pharmacokinetics in postmenopausal women. J Nutr, 132:2587-92.

Rowland I, Faughnan M, Hoey L, et al. 2003. Bioavailability of phytooestrogens. Br J Nutr, 89(Suppl 1):S45-58.

Setchell KD, Clerici C, Lephart ED, et al. 2005. S-equol, a potent ligand for estrogen receptor beta, is the exclusive enantiomeric form of the soy isoflavone metabolite produced by human intestinal bacterial flora. Am J Clin Nutr, 81:1072-9.

Setchell KD, Brown NM, Desai PB, et al. 2003. Bioavailability, disposition, and dose-response effects of soy isoflavones when consumed by healthy women at physiologically typical dietary intakes. J Nutr, 133:1027-35.

Setchell KD, Brown NM, Desai P, et al. 2001. Bioavailability of pure isoflavones in healthy humans and analysis of commercial soy isoflavone supplements. J Nutr, 131(4 Suppl):1362S-75S.
Setchell KD, Borriello SP, Hulme P, et al. 1984. Nonsteroidal estrogens of dietary origin: possible roles in hormone-dependent disease. Am J Clin Nutr, 40:569-78.

Van Erp-Baart MA, Brants HA, Kiely M, et al. 2003. Isoflavone intake in four different European countries: the VENUS approach. Br J Nutr, 89(Suppl 1):815-21.

Vergne S, Titier K, Bernard V, et al. 2007. Bioavailability and urinary excretion of isoflavones in humans: effects of soy-based supplements formulation and equol production. J Pharm Biomed Anal, 43:1488-94.

Xu X, Harris K, Wang HJ, Murphy PA, et al. 1995. Bioavailability of soybean isoflavones depends upon gut microflora women. J Nutr, 125:2307-15.

Xu X, Wang HJ, Murphy PA, et al. 1994. Daidzein is a more bioavailable soymilk isoflavone than is genistein in adult women. $J$ Nutr, 124:825-32. 